Supporting Information

\title{
Nondestructive In Situ Detection of Chemical Reactions at the Buried Interface between Polyurethane and Isocyanate-Based Primer
}

Shuqing Zhang, ${ }^{1}$ John S Andre, ${ }^{2}$ Lorraine Hsu, ${ }^{3}$ Amy Toolis, ${ }^{3}$ Samuel L Esarey, ${ }^{3}$ Bolin Li, ${ }^{2}$ Zhan

$$
\text { Chen }^{* 1,2}
$$

${ }^{1}$ Department of Macromolecular Science and Engineering, ${ }^{2}$ Department of Chemistry, University of Michigan, 930 North University Avenue, Ann Arbor, Michigan 48109, United States

${ }^{3}$ Coatings and Innovation Center, PPG, 4325 Rosanna Drive, Allison Park, PA 15101, United States

*Corresponding Author: Zhan Chen, zhanc@umich.edu 
Table S1. Compositions of the PPC and the primer studied in this research.

\begin{tabular}{|c|c|}
\hline PPC PR 1547 AMB Part A & \\
\hline Ingredient name & $\%$ \\
\hline 2,2'-dichloro-4,4'-methylenedianiline & $\geq 20-\leq 50$ \\
\hline $\begin{array}{l}\text { Poly(oxy-1,4-butanediyl), } \alpha \text {-hydro- } \omega \text {-hydroxy-, polymer with 1,3- } \\
\text { diisocyanatomethylbenzene }\end{array}$ & $\geq 20-\leq 50$ \\
\hline PPC PR1547 AMB Part B & \\
\hline Ingredient name & $\%$ \\
\hline $\begin{array}{l}\text { 1,4-Butanediol, polymer with 2,4-diisocyanato-1-methylbenzene } \\
\text { and .alpha.- hydro-.omega.-hydroxypoly[oxy(methyl-1,2-ethanediyl)] }\end{array}$ & $\geq 90$ \\
\hline 4-methyl-m-phenylene diisocyanate & $\leq 1.0$ \\
\hline Primer PR 420 Part A & \\
\hline Ingredient name & $\%$ \\
\hline Chlorobenzene & $\geq 20-\leq 50$ \\
\hline 4,4'-methylenediphenyl diisocyanate & $\geq 20-\leq 45$ \\
\hline Isocyanic acid, polymethylenepolyphenylene ester & $\geq 10-\leq 20$ \\
\hline o-(p-isocyanatobenzyl)phenyl isocyanate & $\geq 0.10-\leq 2.3$ \\
\hline methylenediphenyl diisocyanate & $\leq 1.3$ \\
\hline 2,4-dioxo-1,3-diazetidine-1,3-diylbis[p-phenylenemethylene-p-phenylene] & $<1.0$ \\
\hline
\end{tabular}




\begin{tabular}{|l|l|}
\hline diisocyanate & \\
\hline 2,2'-methylenediphenyl diisocyanate & $<1.0$ \\
\hline Isocyanic acid, polymethylenepolyphenylene ester, polymer with .alpha.- & $<1.0$ \\
\hline hydro-.omega.-hydroxypoly(oxy-1,2-ethanediyl) & \\
\hline Primer PR 420 Part B & \\
\hline Ingredient name & $\%$ \\
\hline Trichloroethylene & $\geq 20-\leq 50$ \\
\hline Cyclohexanone & $\geq 20-<40$ \\
\hline Silicic acid, chromium lead salt & $\geq 10-\leq 20$ \\
\hline Talc, not containing asbestiform fibres & $\geq 1.0-\leq 5.0$ \\
\hline Lead & $<0.10$ \\
\hline
\end{tabular}

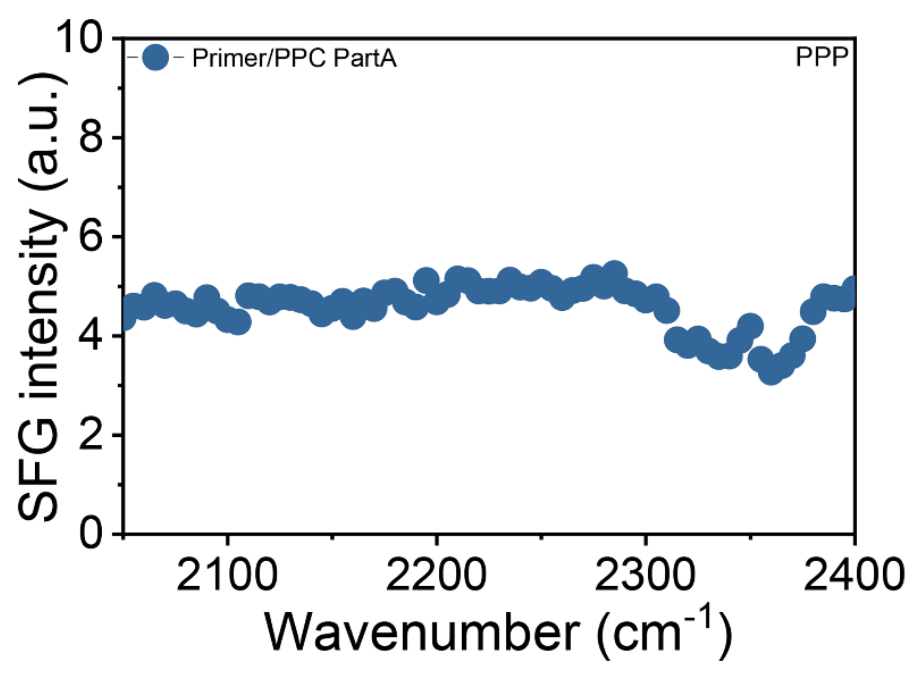


Figure S1. SFG ppp spectrum collected from the primer/PPC Part A interface after annealing. 